\title{
CORRECT SIZING OF REFLECTORS IN ULTRASONIC INSPECTION OF THE FORGING TITANIUM ALLOY
}

\author{
THEODOR TRANCĂ ${ }^{1} \&$ IULIANA RADU ${ }^{2}$ \\ ${ }^{1}$ AROEND, Romania \\ ${ }^{2}$ ZIROM - SA, Romania
}

\begin{abstract}
Commercial Ti-6Al-4V forgings are widely used in the rotating components of aircraft engines. The failure of such parts can be quite catastrophic because of the large amount of kinetic energy. To ensure the safety and longer lifetime of these critical parts working in the hostile environments of high temperature and high stress, the need to detect smaller defects becomes more and more important. Ultrasonic inspection is one of the non-destructive evaluation (NDE) methods widely used by the titanium forging's manufacturers because of its ability to penetrate the interior of a component. Over the last decade, sizing methods were established like DGS (Distance Gain Size) or DAC (Distance Amplitude Correction) for defects smaller than the beam profile. Those methods utilize the echo amplitude and provide results which are proportional to the defect area. In this article, the correct sizing of small defects below one wavelength is investigated. By properly choosing the simulation method, it is ensured that all physical wave modes are included in the simulation and that the discretization error is negligible. A good correspondence between the simulation and classical defect sizing for defects larger than one wavelength is found. In the region between one quarter of a wavelength and one wavelength resonance effects are found, which results in classical defect sizing methods giving conservative results. In the region below one quarter of a wavelength classical DGS and DAC sizing leads to under-sizing.

Keywords: simulation program, grain noise, small defect.
\end{abstract}

\section{INTRODUCTION}

With the improvement of material technology and ultrasonic inspection, the necessity to detect and size smaller defects is higher. Therefore, not only for flat bottom holes, but also for the disc shaped reflectors the usability for small irregularities needs to be checked.

Measurement of ultrasonic indication is generally performed by echo dynamic or area amplitude based sizing. For discontinuities larger than the beam spread, echo dynamic sizing (sizing by probe travel, e.g. $-6 \mathrm{~dB}$ drop method) are practiced and for indications more minor than the beam spread area-amplitude based sizing procedures are applied (like DAC or DGS).

\section{CORRECT DIMENSIONING OF THE REFLECTORS SMALLER THAN ONE WAVELENGTH}

Area-amplitude based sizing procedures compare the reflection of an indication to the reflection of reflectors with known dimension, e.g. artificial reflectors like flat bottom holes (FBH) or side drilled holes (SDH). The most traditional procedures used from the first days of the ultrasound testing, is the Distance Amplitude Correction (DAC), based on calibration managing multiple flat bottom holes machined into calibration blocks and some extrapolation based on the inverse square law.

\subsection{Disagreement with area-amplitude relationship}

The area-amplitude relationship is a consequence of using the Kirchhoff (also called physical optics) approximation when solving the equations governing ultrasound scattering by an FBH. This approximation assumes that the motion of the FBH surface when reflecting 
an ultrasound pulse is identical to the motion that would occur if the pulse were reflecting from an infinite planar surface. A further assumption in obtaining the area-amplitude relationship is that the finite width ultrasound beam can be approximated as an infinite plane wave. Under these assumptions, it is seen that the FBH surface motion will be independent of the size of the FBH. Auld's reciprocity formula [1] states that the voltage received from a void defect is the integral over the defect surface of the product of the traction generated by the incident pulse in the absence of the defect, multiplying the total surface motion of the defect in response to the incident pulse. In the case of the FBH using the Kirchhoff approximation with an incident plane wave, it is readily seen that Auld's formula predicts an output voltage in direct proportion to the area of the $\mathrm{FBH}$, i.e., the area amplitude relationship.

The relationship between echo response and FBHs has been addressed since some of the earliest developments in ultrasonic testing. Krautkrämer [2] referred to these as a disk-shaped reflector (DSR) and developed the famous AVG (English DGS) method of relating amplitude responses from FBHs to curves made for each style of probe. The relationship between echo amplitude and probe and FBH size can be summarised in the form of an equation:

$$
\frac{V_{f}}{V_{0}}=\frac{S A}{\lambda^{2}} \frac{1}{T^{2}} e^{-2 T \delta},
$$

where:

$V_{f}=$ the maximum amplitude of the echo from the target

$V_{0}=$ the maximum possible signal amplitude if all energy is returned to the receiver

$T=$ the distance along the beam axis to the target

$A=$ the area of the defect

$S=$ the area of the probe

$\lambda=$ the wavelength of ultrasound (nominal)

$\delta=$ the attenuation coefficient.

From the findings of Krautkrämer [2], it was possible to note that the amplitude change for a FBH was directly proportional to its area. Therefore, having set a response on the Cathodic Ray Tube (CRT) to a specific amplitude (within the linear region of the instrument display) the response from area would produce a signal with half the amplitude and the response from an FBH double the area would produce a signal with double the amplitude.

As part of producing setup standards, a study was performed to determine if smaller FBH could be used by applying the theoretical gain difference in $\mathrm{dB}$ based on the area amplitude relationship. There are advantages to using biger FBHs since they can be drilled deeper and provide separation between the back-wall signal and the signal from the bottom of the hole.

The results of the Ultrasonic Testing measurements, however, indicated that a smaller difference was consistently observed rather than the theoretical $\mathrm{dB}$ difference (see Fig. 1).

The difference between the signals of $\Phi 2.0 \mathrm{~mm} \mathrm{FBH}$ and $\Phi 1.2 \mathrm{~mm} \mathrm{FBH}$ is measured at $7.5 \mathrm{~dB}$ instead of $8.87 \mathrm{~dB}$, the theoretical value (measured on the $\Phi 265.0$ reference block).

The cause of the discrepancy was determined by modeling the interaction of the beam produced by the specific transducers used and the FBHs.

It was shown that interaction between the surface wave that is generated on the FBHs and the incident compression wave can have a constructive interference that raises the amplitude of the smaller FBH. A phenomenon that the classic theory fails to predict is the generation of diffracted waves at the corners of the FBH. Fig. 2 depicts the various diffraction phenomena that occur for a plane compressional wave at perpendicular incidence on the 


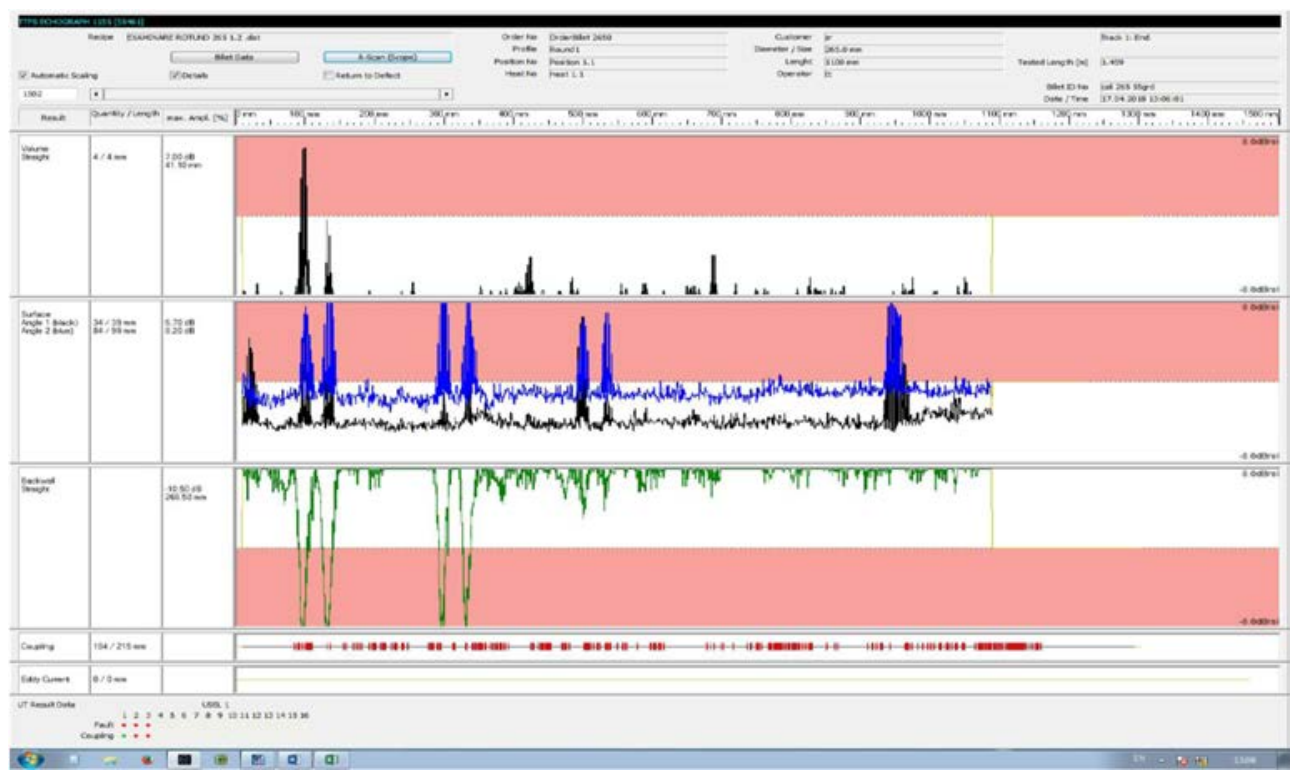

Figure 1: The difference between the signals of $\Phi 2.0 \mathrm{~mm}$ FBH and $\Phi 1.2 \mathrm{~mm} \mathrm{FBH}$.

FBH. It is seen that in addition to a reflected compressional wave, diffracted compressional and shear waves are generated, along with surface waves that propagate both down the bore of the FBH and across the top. Attention is directed to the surface waves propagating across the top of the FBH. Upon reaching the opposing corner of the FBH surface, the surface wave undergoes a second diffraction, during which a small amplitude diffracted compressional wave emerges from the FBH corner. Part of this secondary diffracted wave travels up to the transducer, slightly behind the primary compressional wave reflection from the FBH surface, as depicted in Fig. 3, and is received as a small signal trailing the main reflection, as depicted in Fig. 4. The time delay between these two waves is given by the product of the FBH diameter and the surface wave velocity.

If the time delay between these two signals is sufficiently small, an interaction could take place that would enhance or reduce the total signal amplitude through a constructive or destructive interference. Such an interaction might be the underlying cause of the deviation from the area-amplitude relation seen in experiments when looking at small reflectors.

The challenge in determining the significance of the surface wave interaction is quantitatively determining the amplitudes of the diffracted signals. For this purpose, a computer model was employed that used a boundary element method (BEM) formulation to solve the equations governing the surface wave diffraction phenomena on the FBH. The boundary element formulation uses a high-frequency computational ansatz based on an asymptotic analysis of the diffraction problem. Rather than using the asymptotic solution outright, this method uses the asymptotic result as a starting point, then seeks to find corrections to the asymptotic solution to obtain an exact numerical solution. The boundary elements are, therefore, used to compute corrections to the asymptotic solution, rather than represent the entire solution. Consequently, extremely large problems can be treated with practical computational efficiency. Boundary elements are prescribed over the top and sides of the FBH. The FBH is assumed infinitely long, and the medium is prescribed to have a 
small ultrasonic attenuation, so that the wave field effectively decays to zero after some distance along the FBH. This attenuation is made just small enough so that its presence is not noticed in the computed transducer response signals.

Using the boundary element formulation, the surface motions are computed on the FBH for a very high frequency, very broadband plane wave. The signals from one such computation can be used to predict the response for any signal with a centre frequency within the bandpass of the computation, or equivalently, for any size FBH for an incident pulse of a given frequency, through appropriate filtering and scaling.

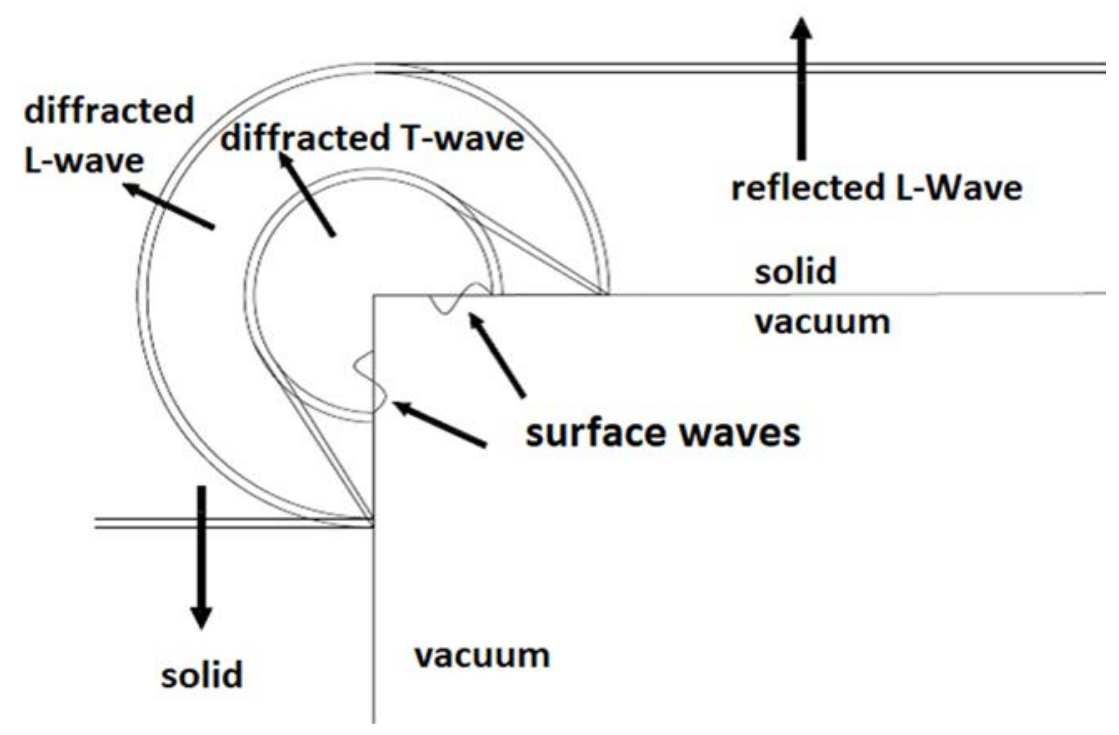

Figure 2: Wave modes generated when a compressional L-wave is incident on an $\mathrm{FBH}[3]$.

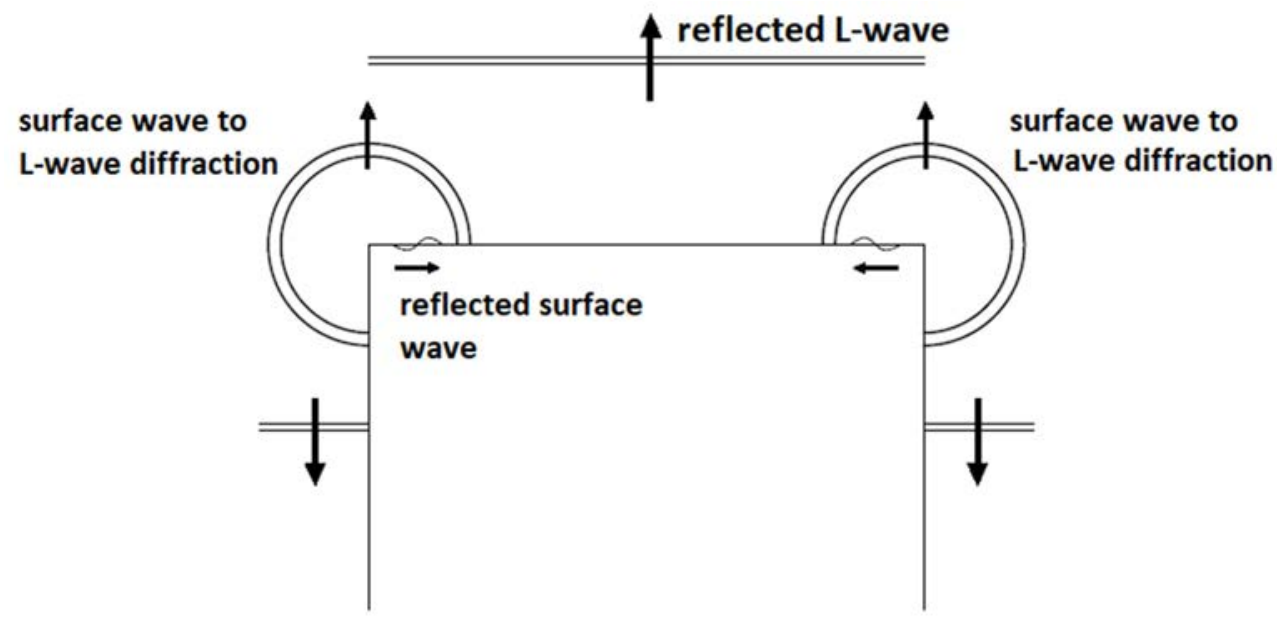

Figure 3: Refracted L-wave following the reflected L-wave [3]. 


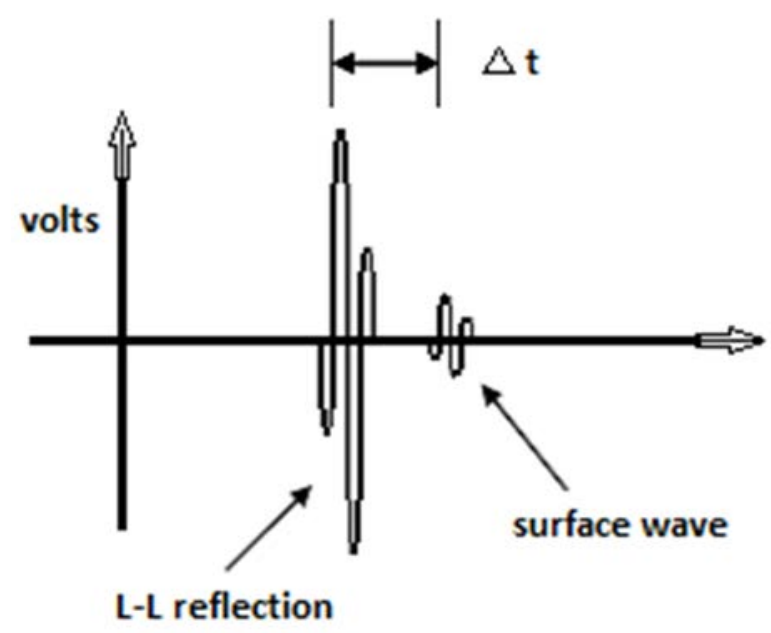

Figure 4: Two signals returned to the transducer [3].
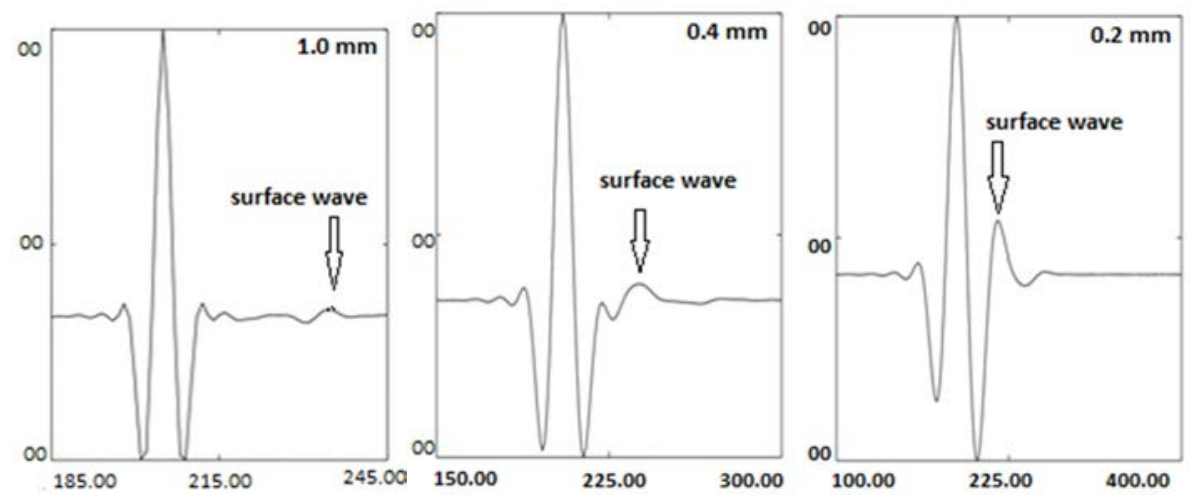

Figure 5: Interaction of the diffracted surface wave and the reflected L-wave for three FBH sizes [3].

Fig. 5 compares signals for $\Phi 1.0 \mathrm{~mm}, \Phi 0.4 \mathrm{~mm}$, and $\Phi 0.2 \mathrm{~mm}$ FBHs. It is seen that as the hole becomes smaller, the time delay between the two received signal components decreases. In the case of the $\Phi 0.2 \mathrm{~mm} \mathrm{FBH}$, the two signals are overlapping. The interaction between the two signals will depend on signal bandwidth: the narrower the bandwidth, the more significant the interaction.

\section{SIMULATION SOFTWARE FOR NDT}

In the simulation used for ultrasonic examination of the planar defects, two classical scattering models have been used: Kirchhoff approximation, to simulate the reflection, and Geometrical Theory of Diffraction, to simulate the diffraction. Recently, from the combination of these two theories, the Physical Theory of Diffraction (PTD) has appeared, which retains the advantages of both. 
It is important to know the boundaries of such simulation tool to be able to assess the simulation results presented in a technical justification. Parameter studies can be used for review of important parameters, in order to find out limit values as well as which parameters are most important for the inspection system. In addition, optimization of defect content for the manufacture of test blocks can be done.

\subsection{Principle of the Kirchhoff and Geometrical Theory of Diffraction model}

Every one of the classical ultrasonic inspection methods, (pulse echo, tandem or Time of Flight Diffraction) perform the detection of the planar defects by interpreting their specular or diffraction echoes. The modern Non-Destructive Testing modelling is useful to assessing detection capability and to elaborate the procedures for inspections.

It assumes that the Kirchhoff scattered field can be decomposed in an approximate manner in two parts: a geometrical field which includes the specular reflected field and a contribution arising from the flaw edges corresponding to the edge's diffraction field. The contribution of this diffraction field at the observation point $\mathrm{x}$ is characterized by same form as the GTD field but a different edge diffraction coefficient (depending on the $\alpha$ incidence and $\beta$ observation directions and polarizations):

$$
U^{K A(D i f f)}(x)=D_{\alpha \beta}^{K A}(x) \frac{e^{i k r}}{\sqrt{k r}}
$$

Note that this coefficient defines the directivity of edge diffraction contribution according Kirchhoff approximation.

The physical theory of diffraction (PTD) consists in correcting the Kirchhoff edge diffraction field by that modelled by GTD. This correction leads to add a corrective term to the KA scattered field (without far-field approximation). This corrective term is the difference of wave amplitudes diffracted by the edge, by GTD and KA.

$$
U^{P T D}(x)=U^{K A}(x)+\left[D_{\alpha \beta}^{G T D}(x)-D_{\alpha \beta}^{K A}(x)\right] \frac{e^{i k r}}{\sqrt{k r}} .
$$

The PTD field is the sum of the Kirchhoff field and a GTD modified field in which the GTD coefficient has been replaced by the difference between GTD and Kirchhoff edge diffraction coefficients. At the specular observation direction, the Kirchhoff field (without far-field approximation) is finite leading to an effective prediction of specular reflection. But the KA diffraction coefficient $\mathrm{KA}, D_{\alpha \beta}^{K A}(x)$ for edge diffraction contribution (previously obtained from a far field approximation of the Kirchhoff field) diverges and has the same singularity as the GTD edge diffraction coefficient GTD, $D_{\alpha \beta}^{G T D}(\mathrm{x})$. When making the difference of the two coefficients, their singularities cancel each other and the diffraction coefficients difference $D_{\alpha \beta}^{G T D}(\mathrm{x})-D_{\alpha \beta}^{K A}(x)$ is finite

$$
U^{P T D}(x) \approx U^{K A}(x) \text {. }
$$

When the observation direction is far from to the specular direction, edge diffraction effects are predominant compared to reflection phenomena, the Kirchhoff field is equal to the Kirchhoff edge diffraction contribution and so cancels it so that the Kirchhoff and GTD model leads to similar results than the GTD model [4]

$$
U^{K A}(x) \approx D_{\alpha \beta}^{K A}(x) \frac{e^{i k r}}{\sqrt{k r}} \text { and } U^{P T D}(x) \approx D_{\alpha \beta}^{G T D}(x) \frac{e^{i k r}}{\sqrt{k r}}=U^{G T D}(x) .
$$


Flaws which can be modelled thanks to Kirchhoff and GTD are the same than with the GTD model: planar flaws (rectangular, semi-elliptical or CAD contour planar flaws), multifacetted flaw and branched flaw [4].

Fig. 6 shows where Kirchhoff and GTD are applicable in relation to the orientation of the defect, with $\alpha=0$ representing a vertical flaw. This figure effectively illustrates what is described in para 3.1. The Kirchhoff approximation in CIVA gives rise to a tip response that is positioned correctly in terms of time of flight, but the amplitude may be inaccurate, with the error increasing with departure of the scatter direction from specular [5].

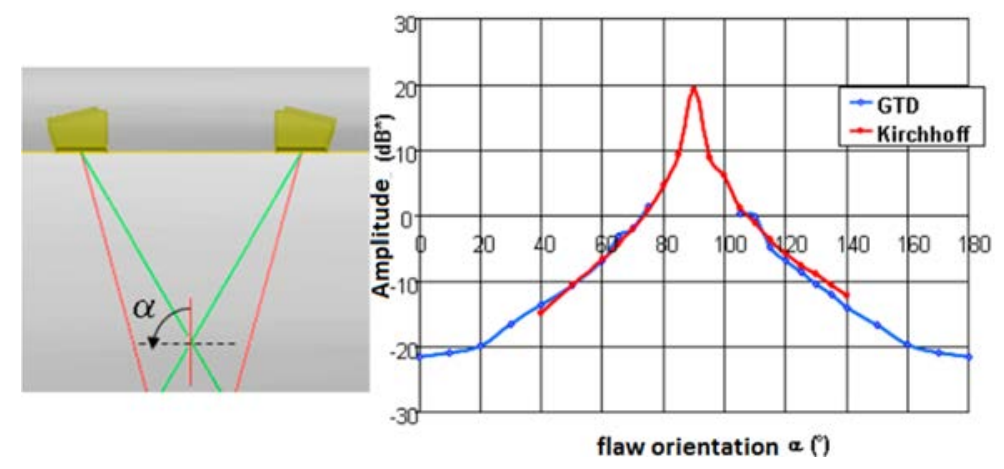

Figure 6: Applicability of Kirchhoff and GTD models.

\subsection{Model capability to be assessed}

The model verification activities are divided into five different partial phases. Each phase is divided into a number of different tasks with specific purposes (see Table 1).

Table 1: Phases of the verification activities.

\begin{tabular}{|l|l|}
\hline Phase 1 & $\begin{array}{l}\text { Response prediction for simple/smooth defects in simple materials and probe } \\
\text { modeling }\end{array}$ \\
\hline Phase 2 & Geometry handling with model \\
\hline Phase 3 & Complex materials - austenitic welds, inconels, dissimilar metal welds \\
\hline Phase 4 & Rough defects in simple materials \\
\hline Phase 5 & Rough defects in complex materials \\
\hline
\end{tabular}

The difference between simple/smooth defects and rough defects stated in Table 1 is that simple/smooth defects are typically artificial defects or an ideal fatigue crack. Rough defects are the type of defects that are typically service induced, with a clear morphology, following grain structure or other irregularities. By simple materials means carbon steel or stainless parent material that shows isotropic behaviour. Complex materials show anisotropic behaviour with significant influence on the sound beam giving effects such as large scattering, beam deflection and increased noise. The noise caused by the material structure is modelled as a separate layer which is super positioned on top of the defect response simulation, meaning that the defect response is not affected by the noise. If a noise simulation is used, it must be used together with additional attenuation modelling as mentioned above or else the result will be a non-conservative signal to noise ratio for any give indication [5]. 


\section{CIVA 11 APPLICATIONS IN THE ULTRASONIC EVALUATION OF THE EXAMINATION STANDARDS}

\subsection{Determination of the FBH's ultrasonic responses}

The goal of this virtual determination is to measure the difference $(\mathrm{dB})$ between the signals obtained from Flat Bottom Holes of different diameters positioned at the same depth into the examined material. The obtained values will be compared with the theoretical difference, calculated according to the Kirchhoff Approximation.

The scanning of three reference blocks with the followings dimensions has been performed:

- $\quad$ Flat faced reference block, 111 x $111 \mathrm{~mm}$ square dimension (Fig. 7),

- $\quad$ Round reference block, $111 \mathrm{~mm}$ diameter (Fig. 8),

- $\quad$ Round reference block, $265 \mathrm{~mm}$ diameter (Fig. 9).

Each reference block is provided with one pair of FBH having the diameters of $\Phi 1.2$ and $\Phi 2.0 \mathrm{~mm}$, first determination, and $\Phi 0.8 \mathrm{~mm}$ and $\Phi 1.2 \mathrm{~mm}$, second determination. All holes are positioned with the flat surface at $50 \mathrm{~mm}$ below the entry surface of the ultrasonic beam.

The ultrasonic transducers used for these simulations are the following:

STS 20 P5 - immersion transducer, non-focused, $20 \mathrm{~mm}$ crystal diameter, $5 \mathrm{MHz}$ central frequency.

STS 20 P5 L125 - immersion transducer, $125 \mathrm{~mm}$ focal distance in water, $20 \mathrm{~mm}$ crystal diameter, $5 \mathrm{MHz}$ central frequency.

STS 20 P5 L200 - immersion transducer, $200 \mathrm{~mm}$ focal distance in water, $20 \mathrm{~mm}$ crystal diameter, $5 \mathrm{MHz}$ central frequency.

Sound path in water is set $100 \mathrm{~mm}$ for all situation. Sound speed in material and specific attenuation are identical for all determinations (see Figs. 7-9).

Each reference block was scanned successively with each of the three transducers, respectively 18 measurement for the pair $\Phi 2.0-\Phi 1.2 \mathrm{~mm} \mathrm{FBH}$ and similarly for the pair $\Phi$ $1.2-\Phi 0.8 \mathrm{~mm}$ FBH. The results of the test are presented in Tables 2 and 3 (see below).

These figures present, in the left side, the echo-dynamic registration of the echoes obtained by scanning each of the two FBH. In the same time, the amplitude of the echoes and difference between signals from the holes can be direct read. In the right side of the pictures is represented the examination technique related to the reference block used and the holes positions.

\section{RESULTS}

\subsection{Comparison between signal's amplitude}

We observe that, for the FBH pair of $\Phi 1.2-\Phi 2.0 \mathrm{~mm}$, the amplitude differences obtained with CIVA 11 are in the range of $8.6 \div 8.8 \mathrm{~dB}$, very close of the theoretical value of $8.87 \mathrm{~dB}$ (see Table 2). Similarly for the FBH pair of $\Phi 0.8-\Phi 1.2 \mathrm{~mm}$, where the difference determined by the program are in the range of $6.8 \div 7.0 \mathrm{~dB}$ and the calculated value is $7.04 \mathrm{~dB}$ (see Table 3). 


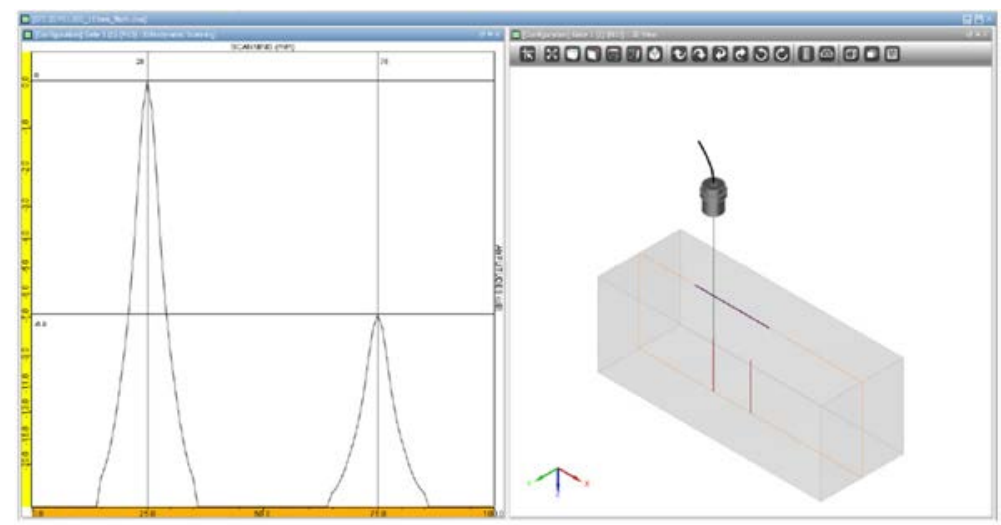

Figure 7: Scanning of the flat-faced $111 \times 111 \mathrm{~mm}$ billet.

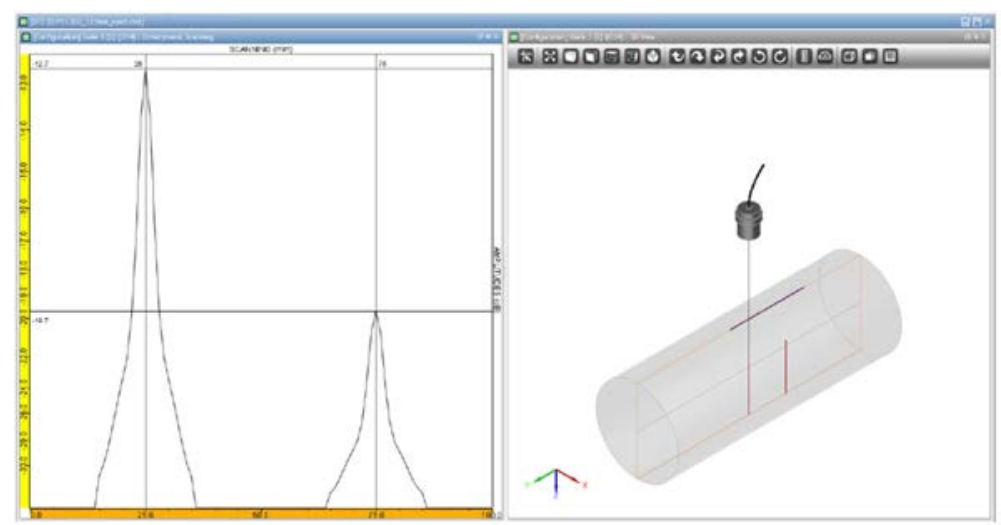

Figure 8: Scanning of the $\Phi 111 \mathrm{~mm}$ billet.

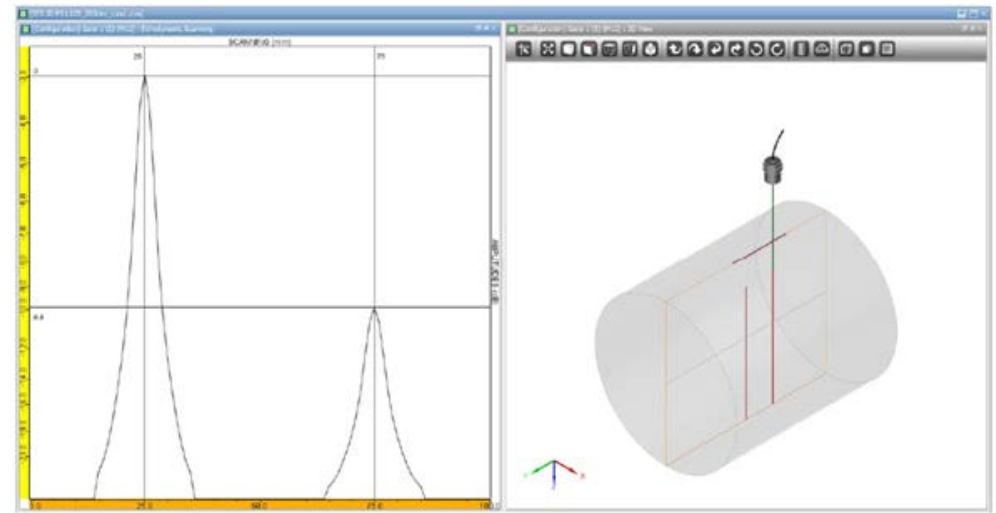

Figure 9: Scanning of the $\Phi 265 \mathrm{~mm}$ billet. 
Table 2: Comparison of difference between signals of FBHs $\Phi 1.2$ and $\Phi 2.0 \mathrm{~mm}$.

\begin{tabular}{|c|c|c|c|c|c|c|c|}
\hline \multirow{3}{*}{$\begin{array}{l}\text { Test } \\
\text { specimen } \\
\text { billet } \\
111 \times 111 \\
\mathrm{~mm}\end{array}$} & \multirow{2}{*}{$\begin{array}{l}\text { Test } \\
\text { flaw } \\
\text { FBH } \varnothing \\
1.2 \mathrm{~mm}\end{array}$} & \multicolumn{2}{|c|}{ STS 20 P5 } & \multicolumn{2}{|c|}{ STS 20 P5 L125 } & \multicolumn{2}{|c|}{ STS 20 P5 L200 } \\
\hline & & $-12.6 \mathrm{~dB}$ & \multirow{2}{*}{$\begin{array}{c}\Delta=8.7 \\
\mathrm{~dB}\end{array}$} & $-9.4 \mathrm{~dB}$ & \multirow{2}{*}{$\begin{array}{c}\Delta=8.7 \\
\mathrm{~dB}\end{array}$} & $-8.6 \mathrm{~dB}$ & \multirow{2}{*}{$\begin{array}{c}\Delta=8.6 \\
\mathrm{~dB}\end{array}$} \\
\hline & $\begin{array}{l}\mathrm{FBH} \varnothing \\
2.0 \mathrm{~mm}\end{array}$ & $-3.9 \mathrm{~dB}$ & & $-0.7 \mathrm{~dB}$ & & $0 \mathrm{~dB}$ & \\
\hline \multirow{2}{*}{$\begin{array}{l}\text { Bar } \varnothing \\
111 \mathrm{~mm}\end{array}$} & FBH $\varnothing$ & $-30.7 \mathrm{~dB}$ & \multirow{2}{*}{$\begin{array}{c}\Delta=8.8 \\
\mathrm{~dB}\end{array}$} & $-16.8 \mathrm{~dB}$ & \multirow{2}{*}{$\begin{array}{c}\Delta=8.8 \\
\mathrm{~dB}\end{array}$} & $-21.3 \mathrm{~dB}$ & \multirow{2}{*}{$\begin{array}{c}\Delta=8.7 \\
\mathrm{~dB}\end{array}$} \\
\hline & $\begin{array}{l}\text { FBH } \varnothing \\
2.0 \mathrm{~mm}\end{array}$ & $-21.9 \mathrm{~dB}$ & & $-8 \mathrm{~dB}$ & & $-12.6 \mathrm{~dB}$ & \\
\hline \multirow[t]{2}{*}{$\begin{array}{l}\text { Bar } \varnothing \\
265 \mathrm{~mm}\end{array}$} & $\begin{array}{l}\mathrm{FBH} \varnothing \\
1.2 \mathrm{~mm}\end{array}$ & $-22.6 \mathrm{~dB}$ & \multirow{2}{*}{$\begin{array}{c}\Delta=8.8 \\
\mathrm{~dB}\end{array}$} & $-11.6 \mathrm{~dB}$ & \multirow{2}{*}{$\begin{array}{c}\Delta=8.7 \\
\mathrm{~dB}\end{array}$} & $-14.1 \mathrm{~dB}$ & \multirow{2}{*}{$\begin{array}{c}\Delta=8.7 \\
\mathrm{~dB}\end{array}$} \\
\hline & $\begin{array}{l}\mathrm{FBH} \varnothing \\
2.0 \mathrm{~mm}\end{array}$ & $-13.8 \mathrm{~dB}$ & & $-2.9 \mathrm{~dB}$ & & $-5.4 \mathrm{~dB}$ & \\
\hline
\end{tabular}

Table 3: Comparison of difference between signals of FBHs $\Phi 0.8$ and $\Phi 1.2 \mathrm{~mm}$.

\begin{tabular}{|c|c|c|c|c|c|c|c|}
\hline \multirow{3}{*}{$\begin{array}{l}\text { Test } \\
\text { specimen } \\
\text { billet } \\
111 \times 111 \\
\mathrm{~mm}\end{array}$} & \multirow{2}{*}{$\begin{array}{l}\text { Test } \\
\text { flaw } \\
\text { FBH } \varnothing \\
0.8 \mathrm{~mm}\end{array}$} & \multicolumn{2}{|c|}{ TS 20 WB4 } & \multicolumn{2}{|c|}{ STS 20 P5 L 125} & \multicolumn{2}{|c|}{ STS 20 P5 L200 } \\
\hline & & $-7.2 \mathrm{~dB}$ & \multirow{2}{*}{$\Delta=7 \mathrm{~dB}$} & $-7.7 \mathrm{~dB}$ & \multirow{2}{*}{$\Delta=7 \mathrm{~dB}$} & $-6.9 \mathrm{~dB}$ & \multirow{2}{*}{$\Delta=6.9 \mathrm{~dB}$} \\
\hline & $\begin{array}{l}\mathrm{FBH} \varnothing \\
1.2 \mathrm{~mm}\end{array}$ & $-0.2 \mathrm{~dB}$ & & $-0.7 \mathrm{~dB}$ & & $0 \mathrm{~dB}$ & \\
\hline \multirow[t]{2}{*}{$\begin{array}{l}\text { Bar } \varnothing \\
111 \mathrm{~mm}\end{array}$} & $\begin{array}{l}\mathrm{FBH} \varnothing \\
0.8 \mathrm{~mm}\end{array}$ & $-21.9 \mathrm{~dB}$ & \multirow{2}{*}{$\Delta=7 \mathrm{~dB}$} & $-15.2 \mathrm{~dB}$ & \multirow{2}{*}{$\Delta=7 \mathrm{~dB}$} & $-19.7 \mathrm{~dB}$ & \multirow{2}{*}{$\Delta=7 \mathrm{~dB}$} \\
\hline & $\begin{array}{l}\mathrm{FBH} \varnothing \\
1.2 \mathrm{~mm} \\
\end{array}$ & $-14.9 \mathrm{~dB}$ & & $-8.2 \mathrm{~dB}$ & & $-12.7 \mathrm{~dB}$ & \\
\hline \multirow[t]{2}{*}{$\begin{array}{l}\text { Bar } \varnothing \\
265 \mathrm{~mm}\end{array}$} & $\begin{array}{l}\mathrm{FBH} \varnothing \\
0.8 \mathrm{~mm}\end{array}$ & $-14.5 \mathrm{~dB}$ & \multirow{2}{*}{$\Delta=6.8 \mathrm{~dB}$} & $-9.9 \mathrm{~dB}$ & \multirow{2}{*}{$\Delta=6.9 \mathrm{~dB}$} & $-12.3 \mathrm{~dB}$ & \multirow{2}{*}{$\Delta=6.8 \mathrm{~dB}$} \\
\hline & $\begin{array}{l}\text { FBH } \varnothing \\
1.2 \mathrm{~mm}\end{array}$ & $-7.7 \mathrm{~dB}$ & & $-3 \mathrm{~dB}$ & & $-5.5 \mathrm{~dB}$ & \\
\hline
\end{tabular}

The resulting values (noted with $\Delta$ in Table 2 and Table 3 ) match the theoretical values obtained for the proportion of the surfaces of the artificial defects. For defects smaller than the wavelength, it was proven in reality that the differences between the two holes are different that these values (please refer to Fig. 1). As observed, these differences are more pronounced as the product $k a>>1$ ( $k=$ wave number and $a=$ size of the artificial defect).

This proves the limitations of the CIVA 11 simulation software for the defects close to the specular area and geometrical reflex whereas the software applies the Kirchhoff Approximation and does not consider the diffraction phenomenon that appear at the edge of FBH.

\subsection{Optimization of the ultrasonic inspection}

The simulation software can also be used for the selection of the ultrasonic transducers in relation with the examination technique for ultrasonic inspection of immersed plates and forged bars.

Table 2 and Table 3 also provide the data relevant for the efficiency of each type of transducer at a certain depth for a specific radius of the entry surface. 
The transducer with the best ultrasonic behaviour for this scenario can be decided by comparing the observed amplification reserve with the value initially selected for each of the 3 transducers.

For an example, if Table 2, row 1 - billet $111 \times 111 \mathrm{~mm}$ and Table 2, row 2 - BAR $\Phi 111$ $\mathrm{mm}$ are selected:

Row 1, FBH $\Phi 1.2 \mathrm{~mm}$ represents the inspection of a defect located at a depth of $50 \mathrm{~mm}$ below the entry plane surface. The best result is provided by the transducer STS 20 P5 L 200 $(-8,6 \mathrm{~dB})$ due to optimal Pulse Volume for a defect located at a $50 \mathrm{~mm}$ depth related to its focal distance in water and the entry plane surface. The gain differences comparing with the other transducers are relatively small: $0.8 \mathrm{~dB}$ versus STS 20 P5 L 125 and $3.0 \mathrm{~dB}$ versus STS 20 P5 (unfocused).

The situation is significantly different when the $\Phi 111 \mathrm{~mm}$ billet is inspected. The most efficient transducer is STS 20 P5 L 125 with a focal length in water of $125 \mathrm{~mm}$. Due to the radius of the entry surface, the focal distance in the material is increased (see para 6.) and this transducer presents now the focal distance in the area of depth of the artificial defect $(50 \mathrm{~mm})$. The gain difference is increased significantly comparing with the other 2 transducers: $4.5 \mathrm{~dB}$ versus the transducer focalized with the focal distance of $125 \mathrm{~mm}$ and $13.9 \mathrm{~dB}$ versus the unfocalized transducer. This is caused by the defocusing of the immersion transducers at incidence area of the ultrasonic beam with the cylindrical surface of the billet and due to the modification of the Pulse Volume in the area of the reference defect.

\section{CONCLUSION}

The accurate prediction of absolute noise levels requires detailed knowledge of the metal microstructure which enters the model calculations through certain frequency-dependent factors known as "backscatter coefficients" or "Figures-of-Merit". Unfortunately, the largest discrepancy between the software simulations and experiments is noise, or rather signal to noise ratio. Defects responses are often evaluated in relation to the surrounding noise levels rather than an arbitrary reference target, such as a notch or SDH.

The issues discussed above means that it is clear that it is not possible to simulate a complete inspection or validate an inspection procedure by simulations with CIVA at the current time. The conclusion is that simulations using CIVA can be used when specific problems or technical solutions must be solved or developed, e.g. the influence of the surface curvature over the Pulse Volume or the correct choice of the inspection configuration.

The inspection's optimization is achieved today both by minimizing the noise of the material and by adopting the inspection techniques capable to highlight and perform a correct evaluation of the discontinuities smaller than one wavelength, according to the highest quality standards.

\section{ACKNOWLEDGEMENTS}

This work was supported by S.C. ZIROM-S. A - Giurgiu, Romania and was performed by the NDT Consulting Company - DIAC SERVICII srl and AROEND, Romania.

\section{REFERENCES}

[1] Auld, B.A., Acoustic Fields and Waves in Solids, vol. II, Krieger Publishing Company: Malaber, 1990.

[2] Krautkrämer, J., Fehlergrößenermittlung mit Ultraschall. Archiv für Eisenhüttenwesen, 30, pp. 693-703, 1959. 
PI-132 Computational Methods and Experimental Measurements XIX

[3] Margetan, F.J.L. et al., Inspection Development for Titanium Forgings, Air Traffic Organization Operations Planning Office of Aviation Research and Development Washington, DC, 20591, May 2007.

[4] Darmon, M., Dorval, V.\& Kamta Djakou, A., A system model for ultrasonic NDT based on the Physical Theory of Diffraction (PTD). Ultrasonics, 64, pp. 115-127, 2016.

[5] Holmer, G., Daniels, W. \& Zettervall, T., Evaluation of the Simulation Software CIVA for Qualification Purpose, SQC Swedish Qualification Centre, AMEC Foster Wheeler: Birchwood, 2017. 